\title{
Oxytocin and the stress buffering effect of social company: a genetic study in daily life
}

\author{
Maurizio Sicorello, ${ }^{1}$ Linda Dieckmann, ${ }^{1}$ Dirk Moser, ${ }^{1}$ Vanessa Lux, ${ }^{1}$ \\ Maike Luhmann, ${ }^{2}$ Wolff Schlotz, ${ }^{3,4}$ and Robert Kumsta ${ }^{1}$
}

\author{
${ }^{1}$ Department of Genetic Psychology, Faculty of Psychology, Ruhr-University Bochum, 44801 Bochum, Germany \\ ${ }^{2}$ Department of Psychological Methods, Faculty of Psychology, Ruhr-University Bochum, 44801 Bochum, \\ Germany, ${ }^{3}$ Max-Planck-Institute for Empirical Aesthetics, Frankfurt am Main, Frankfurt am Main, Germany \\ and ${ }^{4}$ Institute of Psychology, Goethe University, Frankfurt am Main, Frankfurt am Main, Germany \\ Correspondence should be addressed to Robert Kumsta, Department of Genetic Psychology, Ruhr-University Bochum, Universitätstraße 15044780 \\ Bochum, Germany. E-mail: robert.kumsta@rub.de. \\ Present address: Maurizio Sicorello, Department of Psychosomatic Medicine and Psychotherapy, Central Institute of Mental Health, Medical Faculty \\ Mannheim, Heidelberg University, Germany
}

\begin{abstract}
Social relationships are a crucial determinant of both mental and physical health. This effect is partly due to social buffering of stress. Animal studies suggest that social buffering is mediated via the oxytocin system, while studies in humans are sparse and limited by the low ecological validity of laboratory settings. In the present study, participants $(N=326)$ completed smartphone questionnaires four times a day over 4 to 5 days, measuring stressors, negative affect, and social context to assess social buffering. We found that under stress, participants reported a higher need for social company. Further, the impact of prior stressful events on momentary negative affect was attenuated by the perceived pleasantness of current social company. This social buffering effect was moderated by haplotypes of the oxytocin receptor gene, based on two well-described single nucleotide polymorphisms (rs2268498, rs53576). Effects were robust when controlling for gender and age, applying different data quality criteria, and even apparent in genotype-based analyses. Our findings demonstrate that social buffering and its modulation by oxytocin system characteristics have implications for life as lived outside the laboratory.
\end{abstract}

Key words: social buffering; stress; oxytocin; ecological momentary assessment (EMA); genetics

Homo sapiens is a social species: we organize in groups, cooperate with genetically unrelated individuals (Tomasello, 2014), and mostly live in monogamous pair-bonding relationships (Wellings et al., 2006). From a basic evolutionarily perspective, being social can be highly adaptive, as resources for alertness and defense are shared and risk of falling prey is reduced for the individual (Sorato et al., 2012). Even in modern societies, low quality of social relationships is a crucial determinant of mortality (Holt-Lunstad et al., 2010), markers of cardiovascular disease and immune system function (Umberson and Karas Montez, 2010), as well as stress-related mental disorders like depression (Teo et al., 2013). These conditions produce considerable suffering and enormous costs on healthcare systems, making knowledge on the basic mechanisms underlying the effect of social relationships essential to implement effective health policies (Umberson and Karas Montez, 2010). 
Social buffering is one of the basic mechanisms which explain the effect of relationship quality on health (Cohen, 2004). Under stress, the body undergoes a cascade of physiological responses to maintain biological homeostasis, including the activation of the sympathoadrenal and the hypothalamicpituitary-adrenomedullary (HPA) axes, resulting in the release of (nor-)adrenaline and cortisol into the bloodstream. If stressors are sufficiently frequent, severe, or enduring, they lead to wear and tear on the body which is related to the same health conditions observed in people with low relationship quality (Juster et al., 2010). Social support has been shown to buffer these stress-induced physiological changes in humans (Thorsteinsson and James, 1999). This effect is often attributed to high-level functions, such as granting informational, instrumental, or emotional support (Cohen, 2004). Still, physiological stressbuffering effects are even observed in the mere presence of a conspecific in a variety of species. This has long been taken to suggest that the effect of social support can also operate through a more basic biological pathway (Bovard, 1959; Kikusui et al., 2006; Hostinar et al., 2014).

The oxytocin system is presently the most likely biological substrate to underlie the stress buffering effect of social support (Hostinar et al., 2014). Oxytocin is an evolutionarily highly conserved neuropeptide implicated in social and reproductive processes across different species (Donaldson and Young, 2008). Social interactions can trigger oxytocin release from the paraventricular nucleus of the hypothalamus, attenuating the HPA response in rodents (Smith and Wang, 2014). This buffering effect was also observed in isolated animals after central oxytocin administration and could be eliminated with an oxytocin antagonist (Smith and Wang, 2014). Moreover, several studies confirmed with opto- and chemogenetic methods that oxytocinergic activity causally attenuates the fear response (Hasan et al., 2019; Grund et al., 2019; Knobloch et al., 2012). Still, the validity of animal studies for human behavior must be interpreted with caution, as oxytocin can influence stress buffering differently dependent on species and developmental stage (Donaldson and Young, 2008; Hostinar et al., 2014), requiring the validation in humans.

In humans, three laboratory studies have confirmed oxytocin's role in social buffering. Firstly, the combination of social support and intranasal oxytocin led to a prominent reduction of the cortisol response after the Trier Social Stress Test above the effects of social support or oxytocin administration alone (Heinrichs et al., 2003). Secondly, a single nucleotide polymorphism (SNP) in the oxytocin receptor gene was associated with reduced cortisol responses to psychosocial stress in the same experimental paradigm, with carriers of an adenine instead of a guanine base at locus rs53576 profiting less from social support (Chen et al., 2011). Thirdly, a very recent study tested the effect of intranasal oxytocin and a supportive friend on the response to a virtual Trier Social Stress Test (Riem et al., 2020). They found that participants who received both social support and intranasal oxytocin had the lowest levels of anxiety, cortisol, and autonomic activity, albeit both before and after the stress induction. Interestingly, they also reported that oxytocin led to higher perceived emotional support, raising the possibility that oxytocin's role in social buffering might be due to an increased sensitivity to social context (Shamay-Tsoory and Abu-Akel, 2016). Nevertheless, meta-analyses reported mixed results for the effect of intranasal oxytocin administration on physiological responses to threat, which is a central element in the causal chain of social buffering in animal models (Cardoso et al., 2014; Grace et al., 2018; Leppanen et al., 2018). Moreover, it is unclear whether the reported association between oxytocin and social buffering has implications for psychological functioning outside the laboratory. Bringing oxytocin research to real-life settings is a necessary step to understand social buffering in humans, as the correspondence between laboratory and field studies often remains untested.

We used ecological momentary assessment (EMA) to build person-specific models of social buffering in daily life. Participants received short questionnaires four times a day over several days. This method circumvents problems with retrospective response bias and approximates biological processes more closely than classical self-reports (Conner and Barrett, 2012). Moreover, participants can be assessed in a variety of environmental configurations in a within-person manner, without the need to randomize them into stress/no-stress and support/nosupport conditions. This can be advantageous in terms of statistical power and the elimination of interpersonal confounds introduced by randomization.

So far, most studies which tested the social buffering hypothesis in daily life relied on a single trait measurement of perceived social support, which is likely confounded with many other dispositional and socioeconomic characteristics (Steptoe, 2000; Giesbrecht et al., 2013; Smyth et al., 2014). Therefore, we observed not only stressors and affect, but also social context at every measurement occasion. Specifically, we asked participants whether they were alone during the prompt and how pleasant their current company was. A modulation of stress reactivity by the pleasantness of current company represents social buffering in our study. This operationalization differs from past laboratory experiments where social support was usually operationalized through the presence of a friendly individual versus being alone. The former operationalization was chosen here to accommodate that in daily life not all stress buffering social situations might be with friends and, at the same time, not all interactions with friends are necessarily stress buffering. Having a continuous measure of how pleasant people find their current social environment and testing whose stress reactivity is more strongly attenuated by increasingly pleasant social environments could sidestep the problems created by the complexity of social interactions in daily life.

In a next step, moderation of social buffering by relevant genetic polymorphisms can be used to inform the function of the oxytocin system (Moore and Depue, 2016). The oxytocin receptor gene is the most promising gene in this regard (Kumsta and Heinrichs, 2013; Feldman et al., 2016). We chose two candidate polymorphisms based on the literature. The rs53576 is the most researched oxytocin-related SNP in humans which was previously associated with social buffering in a laboratory study (Chen et al., 2011) and has been implicated in social cognition and behavior more generally (Feldman et al., 2016; Kumsta and Heinrichs, 2013; Li et al., 2015; but also see Bakermans-Kranenburg and Van Ijzendoorn, 2014). Carriers of the A allele have been reported to profit less from social support, trust less, exhibit less sensitive parenting behavior, and have lower empathy (Kumsta and Heinrichs, 2013). It is located in an intronic region of the oxytocin receptor gene (i.e. not transcribed into mature mRNA) and therefore likely marks another functional polymorphism, rather than having a functional effect itself. In contrast, the second SNP, rs2268498, lies in the promotor region of the oxytocin receptor gene and is not only associated with social behavior and cognition (e.g. Melchers et al., 2015; Christ et al., 2016) as well as social network size derived from smartphone data (Sariyska et al., 2018). It has also been shown to influence oxytocin receptor density in the human brain tissue (Reuter et al., 2017) and restingstate functional connectivity between amygdala nuclei and a broad range of brain regions (Zimmermann et al., 2018). 
Instead of separately testing the individual SNPs, we employed a haplotype-based approach (Foulkes, 2009). While genotypes indicate gene variants at a single location on both DNA strands, haplotypes indicate gene variants at different locations on a single DNA strand. This approach builds on two facts. First, SNPs in close proximity on one DNA strand are more likely to be inherited together, leading to linkage disequilibrium, meaning the occurrence of close SNPs is correlated. As a result, for example, knowing a person's rs53576 variant can partially inform their rs2268498 variant. Second, SNPs reported in the literature are often not the functional SNPs of interest but rather mark (i.e. are correlated with) actual SNPs of interest nearby. These functional SNPs are more highly correlated with haplotypes than with the compound SNPs that make up the haplotype (Zaykin et al., 2002), an approach already proven useful for the pharmacogenetics of oxytocin (Chen et al., 2015).

We predicted that (1) the effect of stressors on negative affect is attenuated when people are in more pleasant company and that (2) this social buffering effect is moderated by haplotypes on the oxytocin receptor gene.

\section{Methods}

\section{Participants}

In total, 418 people participated in the EMA study, who were recruited via bulletin boards at the Ruhr-Universität Bochum (Germany). Of these, 326 gave their consent for genetic analyses and were successfully genotyped for both SNPs. A previous laboratory study reported a partial $\eta^{2}$ of 0.03 for the interaction between the rs53576 and social buffering (Chen et al., 2011), which corresponds to a product-moment correlation of $r=0.17$ (Lakens, 2013). A simulation-based sensitivity analysis showed that our design had sufficient statistical power of $(1-\beta)=80 \%$ to detect an effect size of $r=0.04$ for the three-way interaction between stressors, pleasantness of social company, and haplotype (Supplementary Figure S1). This corresponds to 0.16\% variance explained (given uncorrelated predictors), more than one order of magnitude smaller than the original effect (Chen et al., 2011). We further calculated the positive predictive value for different simulated true effect sizes, which indicates the probability that a significant finding is a true positive given our study design (Ioannidis, 2005). Under the assumption of equal prior probabilities for $\mathrm{HO}$ and $\mathrm{H} 1$, the positive predictive value was above $80 \%$ for an effect size of $r=0.02$ (around $0.04 \%$ variance explained). Details on the sensitivity analysis can be found in the supplementary material.

The final sample comprised participants between the ages of 17 and $63(M=25.07, S D=8.62)$ of which most were female (77\%) university students (78\%). Other named occupations were full-time work (33\%), vocational school $(3 \%)$, high school $(2 \%)$, no occupation (1\%), or miscellaneous (1\%; some participants endorsed more than one option). Nine participants never filled in questionnaires when they had social company, reducing the effective sample size for the main analysis to $N=317$. There was no monetary incentive. University students could receive partial course credit. This method section partly overlaps with a previous paper on the same participants (Sicorello et al., 2019) which addressed a distinct research question.

\section{Materials and procedure}

Genotyping. During an initial laboratory session, saliva samples for DNA extraction were collected. Detailed information on the procedure can be found in the supplementary material. For rs $2268498,30 \%$ were $\mathrm{T} / \mathrm{T}, 50 \%$ were $\mathrm{T} / \mathrm{C}$, and $20 \%$ were $\mathrm{C} / \mathrm{C}$ carriers. For rs $53576,8 \%$ were $\mathrm{A} / \mathrm{A}, 46 \%$ were $\mathrm{A} / \mathrm{G}$, and $46 \%$ were $\mathrm{G} / \mathrm{G}$ carriers. No deviation from Hardy-Weinberg equilibrium was observed for both genotypes: rs2268498: $\chi^{2}(1)=0.08, P=0.821$, and rs53576: $\chi^{2}(1)=1.60, P=0.245$. The two SNPs were in strong linkage disequilibrium, $r=0.52, D^{\prime}=0.70, \chi^{2}(1)=176.87, P<0.001$.

EMA. The assessment took place in form of a stratified randomized sampling design over five consecutive days in a first wave $(N=158)$ and four consecutive days in a second wave $(N=168)$ to simplify recruitment. Participants were asked to complete a 5 min survey four times each day, with one random prompt within each of the following fixed intervals: $11.15-12.15 \mathrm{~h}, 14.00$ $15.00 \mathrm{~h}, 17.45-18.45 \mathrm{~h}$, and 21.30-22.30 h. During the four intervals, participants received an e-mail from the online platform 'Unipark' with a link to the EMA questionnaire and the request to answer the questions promptly. Participants were informed about the procedure during the laboratory session. They could delay their response if it was received in a situation where responding was inconvenient. Participants had to possess a smartphone which could notify them when they received the e-mail. They were informed about the importance to honestly fill out as many questionnaires as possible in a timely manner. Compliance was high, with participants returning on average $82 \%$ of the questionnaires ( $S D=23 \%$, range: $10-100 \%$ ).

Nine percent of individual questionnaires were removed because less than $50 \%$ of items were completed or time to the preceding questionnaire was below 15 minutes, as questionnaires were not deactivated after their designated time frame. Hence, the time between two questionnaires could be as short as 16 minutes, which is still within the recommended boundaries in EMA research (Ebner-Priemer and Sawitzki, 2007). These procedures resulted in 4905 usable questionnaires. Of those, 2868 were filled in while in social company, representing the effective number of questionnaires for the main analysis.

Measures. The questionnaires included scales on stressors and negative affect. Momentary negative affect was measured with nine items (see below) from the German version of the Positive and Negative Affect Schedule (Krohne et al., 1996).

Previously, we conducted a two-level exploratory factor analysis for ordered categorical indicators on the items for both positive and negative affect (see the supplements of Sicorello et al., 2019). The analyses suggested the extraction of two factors at both the within- and between-person levels, with acceptable fit indices and clear simple structure after discarding one item. Therefore, this item (exhausted) was not included in the scale value. Reliability estimation for the resulting negative affect scale (distressed; upset; guilty; hostile; irritable; ashamed; nervous; afraid) at the between- and within-person level yielded satisfactory coefficients, indicating high reliability of negative affect measures, $\omega_{\text {within }}=0.80$ and $\omega_{\text {between }}=0.95$. The $\omega$-coefficient is a measure similar to Cronbach's alpha but has many theoretical and statistical advantages (Dunn et al., 2014; Sideridis et al., 2018).

Stressors experienced in the preceding hour were measured with seven items regarding different life domains such as task demands or interpersonal events ('I would rather do something else,' 'I have to make an effort,' 'I was not successful in my activities,' 'Something I have done was devalued by another person,' 'I attended to my tasks only reluctantly,' 'I had a conflict with another person,' 'I completed tasks where I could not afford to make any mistakes'). No factor analysis was conducted on 
Table 1. Descriptive statistics for the self-report measures within and between individuals

\begin{tabular}{|c|c|c|c|c|c|c|c|c|}
\hline Measure & M & $S D_{\text {within }}$ & $S D_{\text {between }}$ & 1 & 2 & 3 & 4 & 5 \\
\hline 1. Negative affect & 11.14 & 9.99 & 8.90 & 1 & $0.67^{* * *}$ & $0.12^{*}$ & $-0.41^{* * *}$ & $0.31^{* * *}$ \\
\hline 2. Stressors & 20.88 & 14.51 & 7.63 & $0.43^{* * *}$ & 1 & 0.04 & $-40^{* * *}$ & $0.35^{* * *}$ \\
\hline 3. Alone & 0.42 & 0.46 & 0.19 & $0.06^{* * *}$ & $0.08^{* * *}$ & 1 & - & - \\
\hline 4. Pleasantness of company & 85.30 & 19.90 & 9.95 & $-0.22^{* * *}$ & $-0.31^{* * *}$ & - & 1 & - \\
\hline 5. Need for company & 24.15 & 25.12 & 16.34 & $0.16^{* * *}$ & $0.13^{* * *}$ & - & - & 1 \\
\hline
\end{tabular}

Note. The correlation matrix shows Pearson correlation coefficients within participants below the diagonal and between participants above the diagonal. Correlations between variables 3 and 5 are not possible, as either variable 4 or 5 was assessed, depending on the response to variable 3 . For the same reason, variables 4 and 5 contain fewer observations than the remaining variables $1-3$.

${ }^{*} P<0.05,{ }^{* *} P<0.01,{ }^{* * *} P<0.001$.

these items, as they capture the occurrence of different mostly independent events which are not necessarily correlated.

At every prompt, participants had to indicate whether they were alone. If they were alone, they had to further indicate whether they would rather like to have company. If they were not alone, they had to indicate how pleasant they find their current company.

Except for the item on whether they were alone, participants responded to all items on a visual analogue scale (range: 0100), with level of agreement as scale anchors. For each measurement occasion, the items for stressors and negative affect were averaged into a single momentary scale value, respectively. Descriptive statistics for the self-report measures within and between individuals are presented in Table 1. Means and standard deviations for self-report measures by genotype can be found in Supplementary Table S1. Correlations between haplotypes, gender, age, and self-report measures can be found in Supplementary Table S2.

\section{Statistical analyses ${ }^{1}$}

The data had a hierarchical structure with measurements (level 1) nested within days (level 2) and nested within participants (level 3). Multilevel analyses were conducted in R (version 3.5.1) using the package 'nlme' to accommodate the dependencies in the data, with a first-order continuous time autoregressive (CAR1) covariance structure on level 1. Level-1 predictors were centered on the person mean, so they represent pure withinperson effects. We included random slopes between participants for level-1 predictors if they significantly improved model fit (Matuschek et al., 2017), which was generally the case for stressors and pleasantness of present company. The random slope for their interaction effect was not included, as this model did not converge.

The effect of the oxytocin receptor gene was tested with a haplotype trend regression (Zaykin et al., 2002), following the procedure and R code described in Foulkes (2009). With two biallelic polymorphisms, four haplotypes exist, TA, TG, CA, and CG, with the first letter indicating the rs2268498 variant and the second letter indicating the rs53576 variant. We reconstructed haplotype probabilities with the expectation maximization-based function 'haplo.em()' of the R package 'haplot.stats' (v1.7.9, Sinnwell, 2018). Then, each individual was assigned the conditional expectation for the four haplotypes, given their genotype, using the 'HapDesign()' function published in Foulkes (2009). This approach creates four variables which indicate the expected

1 Data and analysis code to reproduce the results can be found on: https://osf.io/semzg/?view_only=6251a382144e40b0b7adae832bbbc7a4 number of a given haplotype for each individual. As the procedure estimates the 'expected' number of a haplotype for a given individual, values on these variables do not have to be whole numbers $(0,1,2)$, but can also be real numbers (e.g. 0.05, 0.95, 1.87), incorporating the uncertainty in reconstructing haplotypes in some individuals. The relative frequencies of the estimated haplotypes were $\mathrm{TA}=5 \%, \mathrm{TG}=50 \%, \mathrm{CA}=26 \%$, and $\mathrm{CG}=19 \%$.

The effect of haplotype on social buffering was first assessed with an omnibus test. We compared two models with and without the three-way interactions between haplotypes, stressors, and pleasantness using a likelihood ratio test. Both models included haplotype main effects and two-way interactions between haplotypes, stressors, and pleasantness. Only three haplotype variables were included, as the presence of the fourth haplotype is completely determined by the rest, leading to perfect collinearity. For example, we arbitrarily excluded the CG haplotype. When the other three haplotype variables are zero for an individual, she/he must have two versions of the CG haplotype. Statistical results are identical, regardless of which haplotype variable is omitted.

After the omnibus test, we conducted separate regression with only one of the four haplotype variables in the model, respectively. Equations for these models can be found in the supplemental online material. Model equations for the omnibus test were identical, except they included three instead of one haplotype variable.

To check for robustness, we repeated significant genetic models while controlling for gender and age. In gene-environment interaction studies, appropriate control for covariates involves the inclusion of covariate-environment and covariate-gene interactions (Keller, 2014). To adapt this logic to our threeway interaction scenario, we included the interactions covariate-stressors-pleasantness, covariate-stressors-haplotype $\mathrm{i}_{\mathrm{i}}$, covariate-pleasantness-haplotype $_{i}$ as well as all two-way interactions and the main effects contained in these interactions (i indicates the respective haplotype variable, e.g. the conditional expectation for TC). As our main model included 3 haplotype variables, controlling for 2 covariates adds a total of 26 fixed effects. In the separate regressions with only 1 haplotype per model, it adds 12 fixed effects. This might limit the statistical power of our design and should be interpreted with caution.

\section{Results}

\section{Social buffering}

Negative affect was predicted from stressors, the perceived pleasantness of current social company, and their statistical interaction. Negative affect increased with higher stressor ratings $\left(\gamma_{100}=0.24, \mathrm{SE}=0.02,95 \% \mathrm{CI}=[0.21,0.27], P<0.001\right)$ 

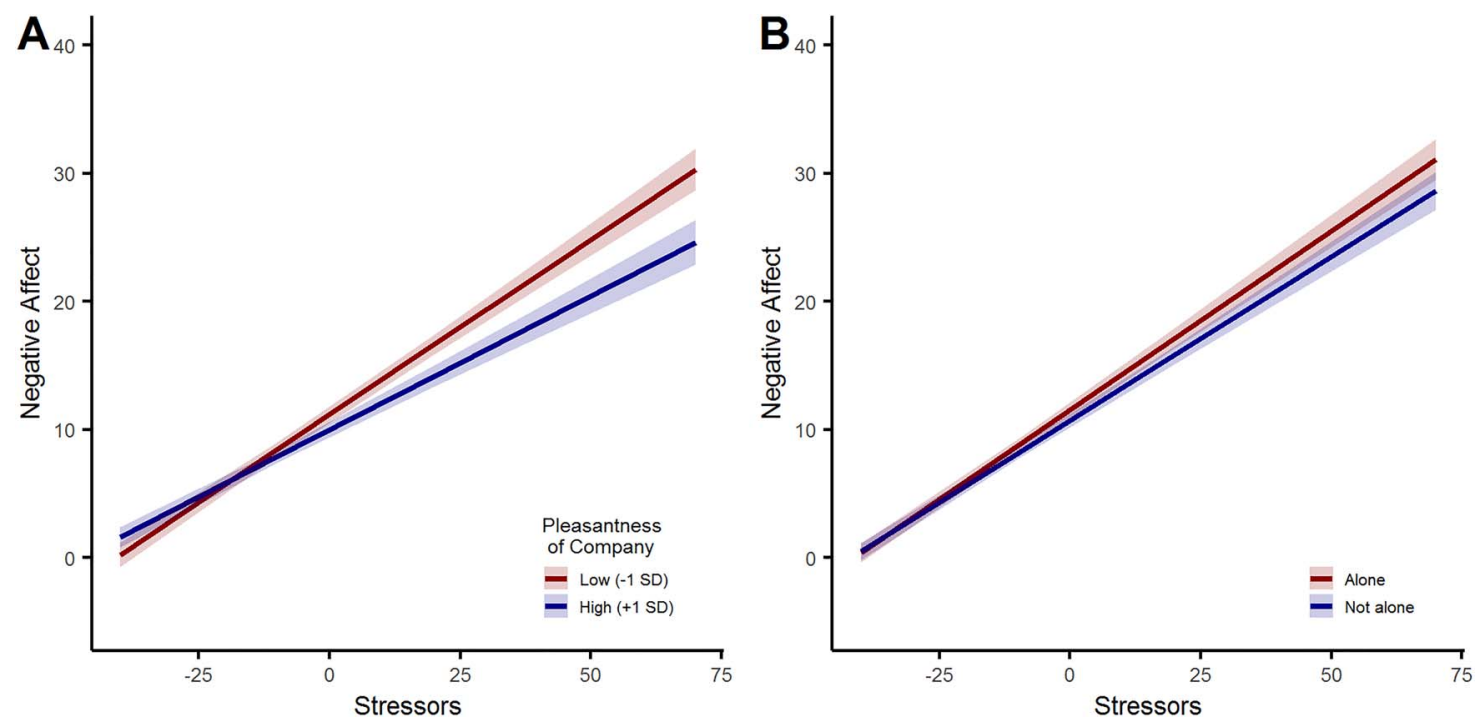

Fig. 1. Effect of social buffering with $95 \%$ confidence bands. (A) Moderation of stress reactivity by pleasantness of current company. (B) Moderation of stress reactivity by being alone.

and slightly decreased with increasing pleasantness of social company $\left(\gamma_{200}=-0.03, \mathrm{SE}=0.01,95 \% \mathrm{CI}=[-0.05,-0.01], \mathrm{P}=0.010\right)$. As hypothesized, the effect of stressors was buffered by pleasantness of social company, marked by a significant interaction $\left(\gamma_{300}=-0.002, \mathrm{SE}=0.001,95 \% \mathrm{CI}=[-0.003,-0.000]\right.$, $P=0.006$ ). For a 10-point increase in pleasantness, the effect of stressors decreased by $7.13 \%$ (Figure 1A).

We assumed that the quality of social company is crucial for social buffering to occur, which has even been shown for non-human species (Kikusui et al., 2006). For example, being among strangers in a train station seems unlikely to buffer against the consequences of prior stressful events. We tested this assumption by including the binary item indicating whether people were currently alone in the model above, instead of pleasantness of social company. As expected, there was no significant interaction between being alone and stressful events (Figure 1B), despite our study being well-powered to detect within-person effects $\left(\gamma_{300}=0.024, \mathrm{SE}=0.019,95 \% \mathrm{CI}=[-0.014\right.$, $0.061], P=0.225)$. There was also no moderation by haplotype $\left(\chi^{2}(3)=3.87, P=0.276\right)$. Being alone was significantly associated with slightly increased negative affect $\left(\gamma_{200}=0.83, S E=0.30\right.$, $95 \% \mathrm{CI}=[0.24,1.41], P=0.006)$, but even the upper limit of the confidence interval indicates only a 1.41-point increase in negative affect when people were alone, representing a practically not meaningful effect size.

\section{Genetic moderation}

Overall, the oxytocin receptor haplotype moderated stress buffering. Model fit improved significantly when the three-way interactions between haplotype, stressors, and pleasantness of current social company were included $\left(\chi^{2}(3)=10.81, P=0.013\right)$. Controlling for gender and age led to similar results $\left(\chi^{2}(3)=8.87\right.$, $P=0.031$ ). In contrast, the statistically not significant interaction between stressors and being alone was not modulated by oxytocin receptor haplotypes $\left(\chi^{2}(3)=3.72, P=0.293\right)$.

We conducted separate regressions for the four haplotypes to further investigate their specific association with social buffering (Foulkes, 2009). Each model tested whether the expected number of copies of a given haplotype moderates the interaction between stressors and pleasantness of current company. We found that in carriers of the CG haplotype, the social buffering effect was significantly less pronounced than in the remaining haplotypes, regardless of whether an uncorrected alpha or a Bonferroni-Holm-corrected alpha was employed (lowest $\alpha=0.013$ ): CG: $\gamma_{301}=0.003, \mathrm{SE}=0.001,95 \% \mathrm{CI}=[0.001$, $0.005], P=0.004$. This effect was practically unchanged and still statistically significant after controlling for gender and age: $\gamma_{301}=$ $0.003, S E=0.001,95 \% \mathrm{CI}=[0.001,0.005], P=0.008$. The effects of other haplotypes were not statistically significant: TG: $\gamma_{301}=$ $-0.001, \mathrm{SE}=0.001,95 \% \mathrm{CI}=[-0.001,0.000], \mathrm{P}=0.073 ; \mathrm{CA}: \gamma_{301}=$ $-0.000, S E=0.001,95 \% \mathrm{CI}=[-0.002,0.001], P=0.607 ;$ and TA: $\gamma_{301}$ $=0.003, \mathrm{SE}=0.003,95 \% \mathrm{CI}=[-0.003,0.008], \mathrm{P}=0.356$ (see Table S3 for additional model output).

Figure 2 depicts the social buffering effect (i.e. the stressor-pleasantness interaction) for groups with one copy of the respective haplotype. Estimates were calculated from the four models reported above by adding the estimate of the haplotypestressor-pleasantness three-way interaction to the estimate of the stressor-pleasantness two-way interaction. The dashed reference line marks the social buffering effect in the model without the haplotype variables, while a value of zero means there is no stress buffering. Note the average social buffering effect is close to the effects of TG and the CA groups, because they made up $75 \%$ of the haplotypes. Effect estimates from the model were multiplied with minus 1 for ease of interpretation, thus more positive values indicate a stronger social buffering effect. The figure again shows that CG carriers exhibited no social buffering effect: Their social buffering effect is not significantly different from zero and slightly goes into the opposite direction, reflecting an exacerbated stress response during pleasant company, albeit with a negligibly small effect size.

Only individuals with a copy of the TG or the CA haplotype exhibited a stress buffering effect significantly different from zero. Interestingly, the estimates of those two haplotypes are practically identical. Their similarity, together with their substantial frequency, explains why neither TC nor CA had a significantly different social buffering effect on its own when compared to the remaining three haplotypes. Notably, the parameter estimate for the TA carriers was similar to the CG group but very 


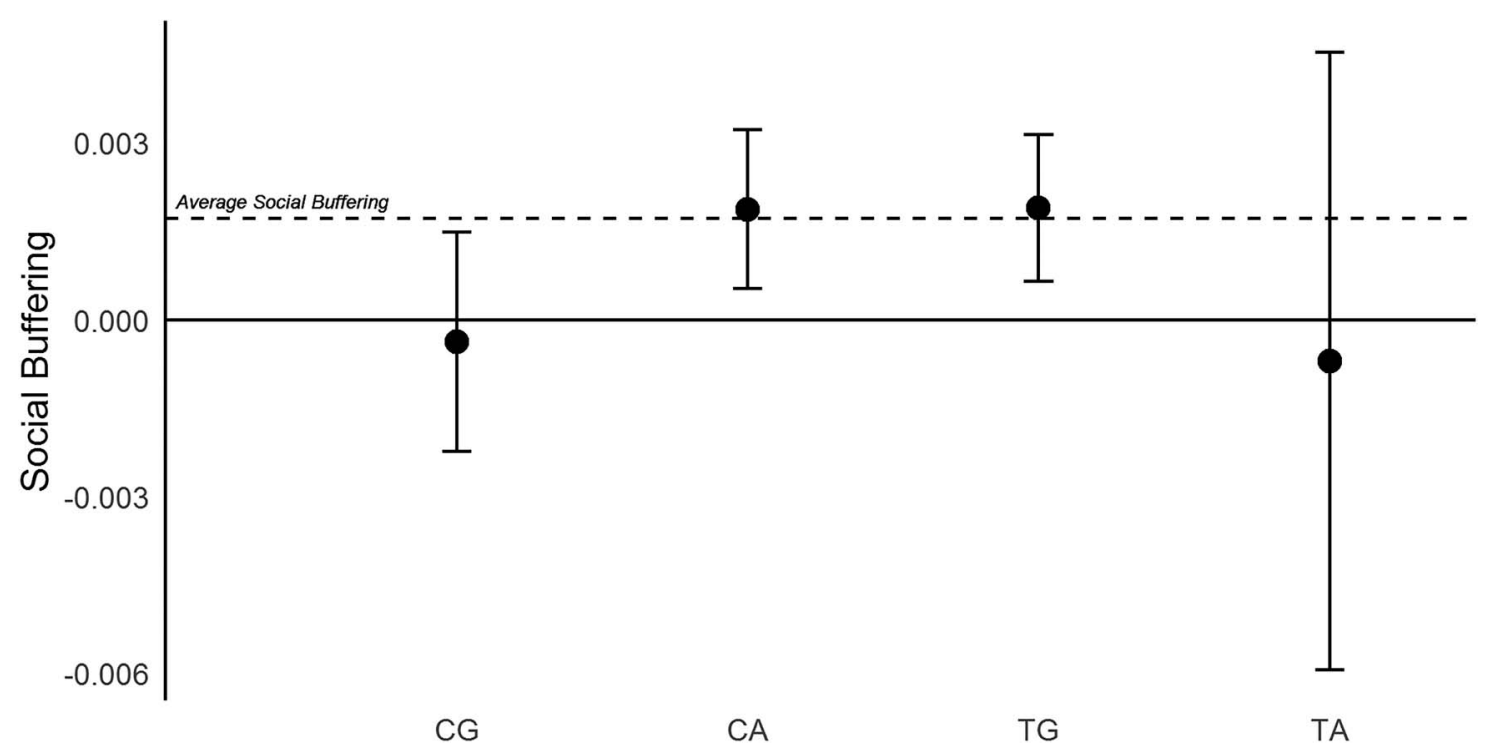

Fig. 2. Social buffering effects for individual haplotypes with $95 \%$ CIs. Large positive values indicate a strong social buffering effect. Social buffering corresponds to the interaction effect of stressors and pleasantness of social company on negative affect. Estimates and standard errors are calculated from regression models and represent the predicted stressors-pleasantness interaction for individuals who have exactly one copy of the respective haplotype.

imprecise as the haplotype was relatively rare, with only 33.29 expected carriers of 1 copy and no carriers of 2 copies.

We repeated the analyses above after employing criteria for careless responding (Meade \& Craig, 2012). We excluded participants who returned less than a third of questionnaires and either had a within-person standard deviation smaller than 1 or a negative within-person Cronbach's alpha on negative affect (Sicorello et al., 2019). This procedure reduced the sample to 275 participants. All results remained identical to those reported above in terms of statistical significance.

Lastly, we tested whether the two SNPs significantly moderated social buffering on their own with a classic genotype approach. We recoded the trichotomous genotype variables into two variables, respectively, using a Helmert regression coding system. Identical to the omnibus test procedure above, we compared a model including genetic three-way interactions against a model with only lower-order terms, separately for the two SNPs. Both SNPs significantly moderated social buffering, even after Bonferroni-Holm correction (lowest $\alpha=0.025$ ): rs2268498: $\chi^{2}(2)=9.38, P=0.009$ and rs53576: $\chi^{2}(2)=6.23, P=0.044$.

\section{Alternative genetic explanations}

The relationship between the oxytocin system, social behavior, and stress processing is complex and still contested (Bartz et al., 2011; Shamay-Tsoory and Abu-Akel, 2016). Therefore, several alternative perspectives on the role of oxytocin exist. First, oxytocin receptor SNPs might directly affect the stressfulness of the environment (gene-environment correlation) or the perceived pleasantness of social interactions. We tested these hypotheses and found that haplotypes did not significantly predict stressors $\left(\chi^{2}(3)=5.70, P=0.127\right)$ or pleasantness of social company $\left(\chi^{2}(3)=0.18, P=0.981\right)$. As the CG haplotype had a significant effect on social buffering, we specifically explored its effect post hoc. The CG haplotype was not associated with the perceived pleasantness of social company $\left(\gamma_{001}=-0.01, S E=1.32\right.$, $95 \% \mathrm{CI}=[-2.60,2.58], P=0.994$; Supplementary Figure S2), but it was associated with the intensity of stressful experiences on an uncorrected alpha level $\left(\gamma_{001}=2.03, \mathrm{SE}=0.90,95 \% \mathrm{CI}=[0.26\right.$, 3.79], $P=0.025$; Supplementary Figure S3). People with one CG copy reported more intense stressors than people without the CG haplotype by 2 points on the 100 -step scale. This analysis was exploratory and conducted after an effect of the CG haplotype on social buffering had been observed on the same data and should be interpreted with caution.

Moreover, the oxytocin system might be related to the need to seek out social support under stress. When our participants were alone, they indicated to what degree they would rather not be alone (range: 0-100). Experiencing stressors increased the need to spend time with other people substantially $\left(\gamma_{100}=0.23\right.$, $\mathrm{SE}=0.05,95 \% \mathrm{CI}=[0.14,0.33], P<0.001)$, but this effect was not modulated by haplotype $\left(\chi^{2}(3)=3.16, P=0.337\right)$.

\section{Discussion}

The oxytocin system is among the most promising targets to understand neural foundations of social behavior and cognition, bearing great potential for both basic and applied psychiatric research. Nevertheless, in humans, the specific role of the oxytocin system in these areas is still highly contested (e.g. Bartz et al., 2011; Shamay-Tsoory and Abu-Akel, 2016). Although animal research offers clear evidence for oxytocin's role in the stress-buffering effect of social support, studies in humans are relatively sparse (Heinrichs et al., 2003; Chen et al., 2011; Riem et al., 2020). Using a combination of genetic and momentary assessment methodology, we show that social buffering of stress-related negative affect occurs in daily life through the pleasantness of social company and is associated with variants of the oxytocin receptor gene. Our findings not only expand basic research on social buffering and confirm the laboratory studies in humans on the modulating effect of oxytocin but most importantly demonstrate that variability in the oxytocin system has implications for life as it is lived outside the laboratory.

In our study, participants expressed a higher need for social company after stressful events. Their response to stressors was attenuated when they had pleasant company, but this social 
buffering effect was absent in carriers of the CG or the TA haplotype, although only the former effect was statistically significant due to the low frequency of TA carriers. Moreover, our data suggests that this effect is robust when potentially careless responders are excluded and gender and age are controlled and cannot be attributed to genetic effects on the perception of social company or a different tendency to seek out social support after stressful events.

We created haplotypes based on two promising candidate polymorphisms. The A allele of the rs53576 has been associated with lower empathy, relative insensitivity to social support, and lower trust (Kumsta and Heinrichs, 2013). For the rs2268498, the $\mathrm{C}$ allele is associated with higher oxytocin receptor density in the human brain tissue (Reuter et al., 2017). Although it could be expected that higher receptor density leads to a higher sensitivity to social stimuli, higher sensitivity is actually observed in carriers of the $\mathrm{T}$ allele (for a discussion see Reuter et al., 2017). In contrast, our haplotype-based pattern suggests that no specific variant on the two SNPs induces a higher sensitivity to social support. Rather, haplotypes containing only one behavioral sensitivity allele-TG or CA-were the only groups which exhibited a social buffering effect. This finding implies effects of unobserved polymorphisms which can be uncovered with a haplotype-based approach. Notably, our haplotype effects do not correspond to the haplotypes which influenced the sensitivity to intranasal oxytocin in the study by Chen et al. (2015), which employed a wider range of SNPs. A replication of our study with their selection of SNPs might be of interest here.

Although we used a (micro-)longitudinal design, it is crucial to note that the data is still correlational in nature. Prior experimental research has demonstrated a causal effect of social support on stress reactivity (Thorsteinsson and James, 1999), but we cannot rule out that stressful environments simultaneously attenuate the positive effect of pleasant social company. Furthermore, regardless of a large age span, our sample consisted mainly of female university students and is therefore not representative of the general public. Also, statistical power is a critical issue in genetic research, as true effect sizes are likely to be small. Our sensitivity analysis attested adequate power to detect an effect size one order of magnitude smaller than the effect size previously reported by the, to our knowledge, only published study on the effect of oxytocin receptor genetics on social buffering in humans (Chen et al., 2011). Moreover, the probability that a significant finding from our design reflects a true positive crosses $80 \%$ when assuming a true variance explained as small as $0.04 \%$. Nevertheless, more research is needed to inform expected effect sizes for complex haplotype interactions in micro-longitudinal studies such as ours. Lastly, our operationalization of social buffering via continuous ratings of the pleasantness of current social context differs from past selfreport studies, which usually employed a single questionnaire of perceived social support, and past laboratory studies, which usually compared the supportive presence of another individual to absence. As one reviewer pointed out, a comparison between pleasant company versus unpleasant and no company would mirror laboratory studies more closely. Because we employed a unipolar rating for pleasantness, we cannot confidently say below which cutoff social company was perceived as unpleasant to test these comparisons. Technically, all ratings above zero should reflect pleasant company. An operationalization which distinguishes between these three groups should be considered in future studies, while we do advocate to also include continuous measures of social context to retain sufficient information on situational valence, as supportive contexts are more complex and multifaceted in daily life. Notably, simply not being alone had no effect on social buffering, which is not surprising, given that social interactions can also be the reason for stressful experiences. Still, it is not completely clear from the data whether people interpreted being among strangers as being alone.

We used two well-researched SNPs, one with proven functional effects, to probe the involvement of the oxytocin system in the responsiveness to social company under real-life stress. This focus on only two SNPs with a strong empirical basis has the advantage of not diminishing statistical power with the correction for a large number of statistical tests. In future studies, predictive power could be increased by following a gene-set approach incorporating multiple variants of genes of theoretical interest, e.g. by applying machine learning and cross-validation techniques to gene variants related to the oxytocin system (Feldman et al., 2016). Given adequate sample size, such approaches could provide a means for theory testing and increased effect sizes. Combining large-scale genetic data with digital phenotypes, created from behavioral smartphone data, might be particularly promising here (Insel, 2017; Montag and Elhai, 2019). This approach can be used to investigate the interaction between social network characteristics and the oxytocin system (Sariyska et al., 2018), which is another central determinant of health, complementary to the concept of social support (Cohen, 2004).

\section{Ethical standards}

The authors assert that all procedures contributing to this work comply with the ethical standards of the relevant national and institutional committees on human experimentation and with the Helsinki Declaration of 1975, as revised in 2008. The authors assert that all procedures contributing to this work comply with the ethical standards of the relevant national and institutional guides on the care and use of laboratory animals. The study was approved by the local Ethics Committee of the Faculty of Psychology, Ruhr-Universität Bochum, and all participants provided written informed consent.

\section{Supplementary data}

Supplementary data are available at SCAN online.

\section{Financial support}

We acknowledge the support by the DFG Open Access Publication Funds of the Ruhr-Universität Bochum.

\section{Conflict of interest}

None declared.

\section{References}

Bakermans-Kranenburg, M.J., Van Ijzendoorn, M.H. (2014). A sociability gene? Meta-analysis of oxytocin receptor genotype effects in humans. Psychiatric Genetics, 24, 45-51. doi: 10.1097/YPG.0b013e3283643684.

Bartz, J.A., Zaki, J., Bolger, N., Ochsner, K.N. (2011). Social effects of oxytocin in humans: context and person matter. Trends in Cognitive Sciences, 15, 301-9. doi: 10.1016/j.tics.2011.05.002.

Bovard, E.W. (1959). The effects of social stimuli on the response to stress. Psychological Review, 66, 267-77. doi: 10.1037/h0045556. 
Cardoso, C., Kingdon, D., Ellenbogen, M.A. (2014). A meta-analytic review of the impact of intranasal oxytocin administration on cortisol concentrations during laboratory tasks: moderation by method and mental health. Psychoneuroendocrinology, 49, 161-70. doi: 10.1016/j.psyneuen.2014.07.014.

Chen, F.S., Kumsta, R., Dvorak, F., et al. (2015). Genetic modulation of oxytocin sensitivity: a pharmacogenetic approach. Translational Psychiatry, 5, e664-7. doi: 10.1038/tp.2015.163.

Chen, F.S., Kumsta, R., von Dawans, B., Monakhov, M., Ebstein, R.P., Heinrichs, M. (2011). Common oxytocin receptor gene (OXTR) polymorphism and social support interact to reduce stress in humans. Proceedings of the National Academy of Sciences of the United States of America, 108, 19937-42. doi: 10.1073/pnas.1113079108.

Christ, C.C., Carlo, G., Stoltenberg, S.F. (2016). Oxytocin receptor (OXTR) single nucleotide polymorphisms indirectly predict prosocial behavior through perspective taking and empathic concern. Journal of Personality, 84, 204-13. doi: 10.1111/jopy.12152.

Cohen, S. (2004). Social relationships and health. The American Psychologist, 59(8), 676-84. doi: 10.1037/0003-066X.59.8.676.

Conner, T.S., Barrett, L.F. (2012). Trends in ambulatory selfreport: the role of momentary experience in psychosomatic medicine. Psychosomatic Medicine, 74, 327-37. doi: 10.1097/PSY.0b013e3182546f18.

Donaldson, Z.R., Young, L.J. (2008). Oxytocin, vasopressin, and the neurogenetics of sociality. Science, 322, 900-4. doi: 10.1126/science.1158668.

Dunn, T.J., Baguley, T., Brunsden, V. (2014). From alpha to omega: a practical solution to the pervasive problem of internal consistency estimation. British Journal of Psychology, 105, 399-412. doi: 10.1111/bjop.12046.

Ebner-Priemer, U.W., Sawitzki, G. (2007). Ambulatory assessment of affective instability in borderline personality disorder: the effect of the sampling frequency. European Journal of Psychological Assessment, 23, 238-47. doi: 10.1027/1015-5759.23.4.238.

Feldman, R., Monakhov, M., Pratt, M., Ebstein, R.P. (2016). Oxytocin pathway genes: evolutionary ancient system impacting on human affiliation, sociality, and psychopathology. Biological Psychiatry, 79, 174-84. doi: 10.1016/j.biopsych.2015.08.008.

Foulkes, A. S. (2009). Applied Statistical Genetics with R. New York, NY: Springer New York. doi: 10.1007/978-0-387-89554-3

Giesbrecht, G.F., Poole, J.C., Letourneau, N., Campbell, T., Kaplan, B.J. (2013). The buffering effect of social support on hypothalamic-pituitary-adrenal axis function during pregnancy. Psychosomatic Medicine, 75(9), 856-62. doi: 10.1097/PSY.0000000000000004.

Grace, S.A., Rossell, S.L., Heinrichs, M., Kordsachia, C., Labuschagne, I. (2018). Oxytocin and brain activity in humans: a systematic review and coordinate-based meta-analysis of functional MRI studies. Psychoneuroendocrinology, 96, 6-24. doi: 10.1016/j.psyneuen.2018.05.031.

Grund, T., Tang, Y., Benusiglio, D., et al. (2019). Chemogenetic activation of oxytocin neurons: temporal dynamics, hormonal release, and behavioral consequences. Psychoneuroendocrinology, 106, 77-84. doi: 10.1016/j.psyneuen.2019.03.019.

Hasan, M.T., Althammer, F., Silva da Gouveia, M., et al. (2019). A fear memory engram and its plasticity in the hypothalamic oxytocin system. Neuron, 1-14. doi: 10.1016/j.neuron.2019.04.029.

Heinrichs, M., Baumgartner, T., Kirschbaum, C., Ehlert, U. (2003). Social support and oxytocin interact to suppress cortisol and subjective responses to psychosocial stress. Biological Psychiatry, 54, 1389-98. doi: 10.1016/S0006-3223(03)00465-7.
Holt-Lunstad, J., Smith, T.B., Layton, J.B. (2010). Social relationships and mortality risk: a meta-analytic review. PLoS Medicine, 7, e1000316. doi: 10.1371/journal.pmed.1000316.

Hostinar, C.E., Sullivan, R.M., Gunnar, M.R. (2014). Psychobiological mechanisms underlying the social buffering of the hypothalamic-pituitary-adrenocortical axis: a review of animal models and human studies across development. Psychological Bulletin, 140, 256-82. doi: 10.1037/a0032671.

Insel, T.R. (2017). Digital Phenotyping JAMA, 318, 1215. doi: 10.1001/jama.2017.11295.

Ioannidis, J.P.A. (2005). Why most published research findings are false. PLoS Medicine, 2, 0696-701. doi: 10.1371/journal.pmed.0020124.

Juster, R.P., McEwen, B.S., Lupien, S.J. (2010). Allostatic load biomarkers of chronic stress and impact on health and cognition. Neuroscience and Biobehavioral Reviews, 35, 2-16. doi: 10.1016/j.neubiorev.2009.10.002.

Keller, M.C. (2014). Gene x environment interaction studies have not properly controlled for potential confounders: the problem and the (simple) solution. Biological Psychiatry, 75, 18-24. doi: 10.1016/j.biopsych.2013.09.006.

Kikusui, T., Winslow, J.T., Mori, Y. (2006). Social buffering: relief from stress and anxiety. Philosophical Transactions of the Royal Society, B: Biological Sciences, 361, 2215-28. doi: 10.1098/rstb.2006.1941.

Knobloch, H.S., Charlet, A., Hoffmann, L.C., et al. (2012). Evoked axonal oxytocin release in the central amygdala attenuates fear response. Neuron, 73, 553-66. doi: 10.1016/j. neuron.2011.11.030.

Krohne, H.W., Egloff, B., Kohlmann, C.-W., Tausch, A. (1996). Untersuchungen mit einer deutschen version der "positive and negative affect schedule" (PANAS) [investigations with a German version of the positive and negative affect schedule (PANAS)]. Diagnostica, 42, 139-56. doi: 10.1037/t49650-000.

Kumsta, R., Heinrichs, M. (2013). Oxytocin, stress and social behavior: Neurogenetics of the human oxytocin system. Current Opinion in Neurobiology, 23, 11-6. doi: 10.1016/j.conb.2012.09.004.

Lakens, D. (2013). Calculating and reporting effect sizes to facilitate cumulative science: a practical primer for t-tests and ANOVAs. Frontiers in Psychology, 4, 863. doi: 10.3389/fpsyg.2013.00863.

Leppanen, J., Ng, K.W., Kim, Y.R., Tchanturia, K., Treasure, J. (2018). Meta-analytic review of the effects of a single dose of intranasal oxytocin on threat processing in humans. Journal of Affective Disorders, 225, 167-79. doi: 10.1016/j.jad.2017. 08.041.

Li, J., Zhao, Y., Li, R., Broster, L.S., Zhou, C., Yang, S. (2015). Association of oxytocin receptor gene (OXTR) rs53576 polymorphism with sociality: a meta-analysis. PLoS One, 10, 1-16. doi: 10.1371/journal.pone.0131820.

Matuschek, H., Kliegl, R., Vasishth, S., Baayen, H., Bates, D. (2017). Balancing type I error and power in linear mixed models. Journal of Memory and Language, 94, 305-15. doi: 10.1016/j.jml.2017.01.001.

Meade, A. W., Craig, S. B. (2012). Identifying careless responses in survey data. Psychological Methods, 17(3), 437-455. doi: 10.1037/a0028085.

Melchers, M., Montag, C., Felten, A., Reuter, M. (2015). The oxytocin receptor gene and social perception. Social Neuroscience, 10, 345-53. doi: 10.1080/17470919.2015.1008646.

Montag, C., Elhai, J.D. (2019). A new agenda for personality psychology in the digital age? Personality and Individual Differences, 147, 128-34. doi: 10.1016/j.paid.2019.03.045. 
Moore, S.R., Depue, R.A. (2016). Neurobehavioral foundation of environmental reactivity. Psychological Bulletin, 142, 107-64. doi: 10.1037/bul0000028.

Reuter, M., Montag, C., Altmann, S., et al. (2017). Functional characterization of an oxytocin receptor gene variant (rs2268498) previously associated with social cognition by expression analysis in vitro and in human brain biopsy. Social Neuroscience, 12, 604-11. doi: 10.1080/17470919.2016.1214174.

Riem, M.M.E., Kunst, L.E., Bekker, M.H.J., Fallon, M., Kupper, N. (2020). Intranasal oxytocin enhances stress-protective effects of social support in women with negative childhood experiences during a virtual trier social stress test. Psychoneuroendocrinology, 111, 104482. doi: 10.1016/j.psyneuen.2019.104482.

Sariyska, R., Rathner, E.-M., Baumeister, H., Montag, C. (2018). Feasibility of linking molecular genetic markers to real-world social network size tracked on smartphones. Frontiers in Neuroscience, 12, 1-6. doi: 10.3389/fnins.2018.00945.

Shamay-Tsoory, S.G., Abu-Akel, A. (2016). The social salience hypothesis of oxytocin. Biological Psychiatry, 79, 194-202. doi: 10.1016/j.biopsych.2015.07.020.

Sicorello, M., Dieckmann, L., Moser, D., et al. (2019). Highs and lows: genetic susceptibility to daily events. PsyArXiv. Doi: 10.31234/osf.io/6m3yr.

Sideridis, G., Saddaawi, A., Al-Harbi, K. (2018). Internal consistency reliability in measurement: aggregate and multilevel approaches. Journal of Modern Applied Statistical Methods, 17. doi: 10.22237/jmasm/1530027194.

Sinnwell, S. (2018). Haplo.Stats: statistical analysis of haplotypes with traits and covariates when linkage phase is ambiguous. R Package Version 179. https://CRAN.R-project.org/package=ha plo.stats.

Smith, A.S., Wang, Z. (2014). Hypothalamic oxytocin mediates social buffering of the stress response. Biological Psychiatry, 76, 281-8. doi: 10.1016/j.biopsych.2013.09.017.

Smyth, J.M., Zawadzki, M.J., Santuzzi, A.M., Filipkowski, K.B. (2014). Examining the effects of perceived social support on momentary mood and symptom reports in asthma and arthritis patients. Psychology \& Health, 29, 813-31. doi: 10.1080/08870446.2014.889139.

Sorato, E., Gullett, P.R., Griffith, S.C., Russell, A.F. (2012). Effects of predation risk on foraging behaviour and group size: adaptations in a social cooperative species. Animal Behaviour, 84, 823-34. doi: 10.1016/j.anbehav.2012.07.003.

Steptoe, A. (2000). Stress, social support and cardiovascular activity over the working day. International Journal of Psychophysiology, 37, 299-308. doi: 10.1016/S0167-8760(00)00109-4.

Teo, A.R., Choi, H.J., Valenstein, M. (2013). Social relationships and depression: ten-year follow-up from a nationally representative study. PLoS One, 8, 1-8. doi: 10.1371/journal.pone.0062396.

Thorsteinsson, E.B., James, J.E. (1999). A meta-analysis of the effects of experimental manipulations of social support during laboratory stress. Psychology \& Health, 14, 869-86. doi: 10.1080/08870449908407353.

Tomasello, M. (2014). The ultra-social animal. European Journal of Social Psychology, 44, 187-94. doi: 10.1002/ejsp.2015.

Umberson, D., Karas Montez, J. (2010). Social relationships and health: a flashpoint for health policy. Journal of Health and Social Behavior, 51, S54-66. doi: 10.1177/0022146510383501.

Wellings, K., Collumbien, M., Slaymaker, E., et al. (2006). Sexual behaviour in context: a global perspective. Lancet, 368, 1706-28. doi: 10.1016/S0140-6736(06)69479-8.

Zaykin, D.V., Westfall, P.H., Young, S.S., Karnoub, M.A., Wagner, M.J., Ehm, M.G. (2002). Testing association of statistically inferred haplotypes with discrete and continuous traits in samples of unrelated individuals. Human Heredity, 53, 79-91. doi: 10.1159/000057986.

Zimmermann, J., Deris, N., Montag, C., et al. (2018). A common polymorphism on the oxytocin receptor gene (rs2268498) and resting-state functional connectivity of amygdala subregions - a genetic imaging study. NeuroImage, 179, 1-10. doi: 10.1016/j.neuroimage.2018.06.014. 